JOURNAL OF

APPLIED

CRYSTALLOGRAPHY

ISSN 1600-5767

\title{
The small-angle neutron scattering instrument D33 at the Institut Laue-Langevin
}

\author{
C. D. Dewhurst, * I. Grillo, D. Honecker, M. Bonnaud, M. Jacques, C. Amrouni, \\ A. Perillo-Marcone,‡ G. Manzin and R. Cubitt
}

Institut Laue-Langevin, BP156, Grenoble 38042, France. *Correspondence e-mail: dewhurst@ill.fr

Received 3 August 2015

Accepted 16 November 2015

Edited by G. Kostorz, ETH Zurich, Switzerland

₹ Current address: CERN, CH-1211 Geneva 23 Switzerland

Keywords: small-angle-neutron scattering (SANS); D33; time-of-flight (TOF); polarization; ${ }^{3} \mathrm{He}$ analysis.

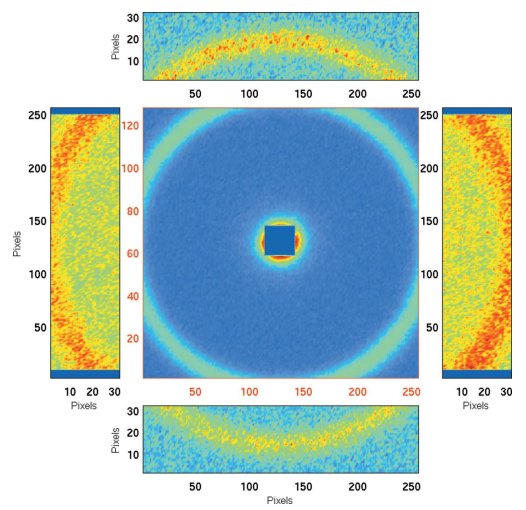

(C) 2016 International Union of Crystallography
The D33 small-angle neutron scattering (SANS) instrument at the Institut Laue-Langevin (ILL) is the most recent SANS instrument to be built at the ILL. In a project beginning in 2005 and lasting seven years, the concept has been developed, and the instrument designed, manufactured and installed. D33 was commissioned with neutrons during the second half of 2012, fully entering the ILL user programme in 2013. The scientific case required that D33 should provide a wide dynamic range of measured scattering vector magnitude $q$, flexibility with regard to the instrument resolution, and the provision of polarized neutrons and ${ }^{3} \mathrm{He}$ spin analysis to facilitate and expand studies in magnetism. In monochromatic mode, a velocity selector and a flexible system of inter-collimation apertures define the neutron beam. A double-chopper system enables a time-of-flight (TOF) mode of operation, allowing an enhanced dynamic $q$ range $\left(q_{\max } / q_{\min }\right)$ and a flexible wavelength resolution. Two large multitube detectors extend the dynamic $q$ range further, giving $q_{\max } / q_{\min } \simeq 25$ in monochromatic mode and a very large $q_{\max } / q_{\min }>1000$ in TOF mode. The sample zone is large and flexible in configuration, accommodating complex and bulky sample environments, while the position of D33 is such as to allow high magnetic fields at the sample position. The instrument is of general purpose with a performance rivalling that of D22, and is well adapted for SANS studies in scientific disciplines as diverse as solution scattering in biology and soft matter and studies of physics, materials science and magnetism. This article provides a detailed technical description of D33 and its performance and characterization of the individual components, and serves as a technical reference for users of the instrument.

\section{Introduction}

Small-angle neutron scattering (SANS) is a well established and powerful technique used to study material or magnetic structures in the size range of about $10-10000 \AA$. The Institut Laue-Langevin's (ILL) suite of small-angle neutron scattering (SANS) instruments, D11, D22 and D33, are often considered as the 'best in the world' in terms of neutron flux at the sample position, with D22 commonly used as a reference bench-mark in the development of new neutron sources and SANS instruments. Applications for SANS beam time at ILL cover a broad scientific base, from solution scattering in biology and soft matter, each representing about one-third of all proposals, to physics, materials science and magnetism, together representing another third. Neutrons scattered by dilute and uniformly shaped particles in solution or dispersed in a material matrix allow the determination of the particle shape function or form factor, particle size distribution, composition and concentration. Concentrated or ordered material structures also provide information concerning particle-particle interactions, crystallographic order, orientation and alignment through the structure and form factors extracted from the recorded scattering pattern (Grillo, 2008; Hollamby, 2013). 
Frequently, complementary techniques such as dynamic light scattering, small-angle X-ray scattering and a host of realspace microscopy techniques are used, with the aim of obtaining a complete analysis of the heterogeneous structural or magnetic system of interest.

It is increasingly required to provide flexible instrument configurations for the optimization of intensity, resolution and dynamic range in scattering vector magnitude $q$. It is also highly desirable to eliminate the need to make two or more independent measurements at different instrument configurations to obtain the overall scattering curve. A time-of-flight (TOF) option using a pulsed 'white' neutron beam allows access to a wide dynamic $q$ range by using a broad band of neutron wavelengths, with TOF SANS instruments typically being found at pulsed spallation neutron sources (Ishikawa $e t$ al., 1986; Seeger \& Hjelm, 1991; Heenan et al., 1997, 2006, 2011). A TOF option is a novel addition to a reactor-based SANS instrument (Kampmann et al., 2006; Dewhurst, 2008), where the pulse frequency and wavelength resolutions can be tailored to requirements. Crucially, this is a situation that cannot easily be achieved with fixed-frequency spallation neutron sources.

Improvements in detector technology allow vast arrays to be built at reasonable cost, as demonstrated by the ILL's monoblock multitube detector technology, arrays of individual position-sensitive tubes and new technologies being developed using solid-state boron-coated wafers. Large detectors allow a large solid-angle coverage and a corresponding wide dynamic $q$ range, important, for example, for many timeresolved measurements of irreversible systems such as crystallization, precipitate formation and critical scattering.

Polarized SANS experiments are becoming increasingly popular owing to intense interest in resolving magnetic structures and dynamics in areas such as ferrofluids (Bonini et al., 2007), magnetic nanoparticles (Disch et al., 2012), nanowire arrays (Grigoryeva et al., 2007), magnetic recording media (Wismayer et al., 2006), microstructure investigation of elemental metals and alloy systems (Heinemann et al., 2000), superconductivity and the vortex lattice (Laver et al., 2006), and chiral and skyrmion-like magnetic structures (Mühlbauer et al., 2009). The insertion of an optional beam polarizer into a SANS instrument must be achieved using a device in transmission, such as a cell of polarized ${ }^{3} \mathrm{He}$ gas, or by reflection out of the beam of the unwanted spin state using a polarizing mirror. Spin analysis of the scattered neutrons can also be made by the temporary installation of a polarized ${ }^{3} \mathrm{He}$ cell analyser (Petoukhov et al., 2006), although careful positioning of the cell next to magnetic sample environments should be considered. One-dimensional (longitudinal or uniaxial) neutron polarization analysis in a SANS experiment has been employed to investigate spin canting in nanoparticle systems (Krycka et al., 2010), anisotropy of the superconducting state in $\mathrm{Sr}_{2} \mathrm{RuO}_{4}$ (Rastovski et al., 2013), magnetization reversal in an exchange-bias system (Dufour et al., 2011), weak ferromagnetism in multiferroics (Ramazanoglu et al., 2011), electric field-induced magnetization in multiferroics (Ueland et al., 2010), magnetostriction in FeGa (Laver et al., 2010) and the (magnetic) microstructure of bulk ferromagnets (Honecker et al., 2010).

This paper describes the scientific design of the D33 instrument and its various components, and their performance and characterization. The overall performance of the instrument is presented, including examples in both monochromatic and TOF modes combined with the use of multiple detectors. The aim of this article is to serve as a technical reference for users of the instrument and the science being performed, as well as for those concerned with the design and construction of future SANS instruments.

\section{Description of instrument components}

Fig. 1 depicts the conceptual design of D33, showing the various neutron optic, beam conditioning and detector components. Briefly, the instrument can be described as a $12.8+12.8 \mathrm{~m}$ instrument, defining the maximum collimation distance and sample-to-detector flight paths. The instrument can be subdivided into four main sections: the casemate section containing most of the optical and beam conditioning components; collimation comprising a $12.8 \mathrm{~m}$ long flight path of either reflecting neutron guide or absorbing collimation tubes and diaphragms; the sample zone accommodating bulky sample environments of up to $2.8 \mathrm{~m}$ along the beam axis; and an evacuated detector tank containing five position-sensitive detector panels mounted on two movable detector trolleys. The individual components and their characterization and performance are discussed in turn below.

\subsection{H14(2) neutron guide}

Neutrons are brought to D33 through the recently renewed $\mathrm{H} 14$ neutron guide system. $\mathrm{H} 14$ begins at the in-pile $\mathrm{H} 1$ insert to the ILL reactor with a direct view of the vertical cold source. The H1 guides provide long-wavelength or 'cold' neutrons to ten instruments on the Vercors side of the ILL7 neutron guide hall. The $\mathrm{H} 14$ guide follows on from the $\mathrm{H} 1 \mathrm{in}-$ pile guide with a cross section of 45 (horizontal) $\times 200 \mathrm{~mm}$ (vertical) and $m=2$ reflective coating, where $m$ is the critical angle for reflection relative to nickel. The first $27 \mathrm{~m}$ of guide curve with radii of $2700 \mathrm{~m}$ (over $6 \mathrm{~m}$ ) and then $4000 \mathrm{~m}$ (over $21 \mathrm{~m}$ ) in the same sense to eliminate direct line-of-sight of the reactor, while opening linearly in the vertical direction to a cross section of 45 (horizontal) $\times 220 \mathrm{~mm}$ (vertical). At this point the single $\mathrm{H} 14$ guide is split into four separate neutron guides, H14(1-4), to supply neutrons to the instruments IN11, LADI, IN12 and D33. The H14(2) guide supplying D33 continues for another $63 \mathrm{~m}$ in a straight line with a cross section of 35 (horizontal) $\times 45 \mathrm{~mm}$ (vertical) and with $m=1$ nickel coating. H14(2) is brought to just inside the D33 instrument blockhouse or 'casemate', which serves as radiological shielding against $\gamma$-radiation produced by the various optical components such as the velocity selector, choppers, polarizers, wavelength filters and attenuators mounted within. The casemate consists of two or three layers of overlapping steel plates of total thickness up to $30 \mathrm{~cm}$, with the thickest 
protection located close to the optical components, such as the velocity selector and polarizers, that create the most $\gamma$-radiation. Neutron guides through the rest of the instrument are made from Borofloat glass with a $30 \times 30 \mathrm{~mm}$ cross section and for the most part are covered with a non-magnetic $m=$ 0.94 nickel-vanadium coating.

\subsection{Beam monitor and beam shutter}

A beam monitor placed directly at the end of the H14(2) guide is the first component in the instrument casemate. The beam monitor is carried by the D33 secondary beam shutter and as such is moved in and out of the neutron beam with the opening and closing of the beam shutter. Since the monitor is before any of the instrument optical components, it measures a fraction of the white neutron flux being delivered by the H14(2) guide. With an efficiency of $\sim 10^{-7}$ and a measured gold-foil beam flux of $1.2 \times 10^{10} \mathrm{n} \mathrm{cm}^{-2} \mathrm{~s}^{-1}$ at the exit of $\mathrm{H} 14(2)$, the monitor has a typical count rate of $\sim 20 \mathrm{kHz}$ and is used to normalize data for fluctuations in the incoming beam intensity due to changes in reactor power.

\subsection{Velocity selector}

A velocity selector provides a quasi-monochromatic source of neutrons with wavelengths between 4.5 and $40 \AA$ and a nominal wavelength spread of $10 \%$ (full width at halfmaximum, FWHM) of triangular distribution. The selector (Astrium EADS) consists of a rotating helical drum of 72 enriched ${ }^{10} \mathrm{~B}_{4} \mathrm{C}$ powder-coated carbon fibre helical blades with a screw angle of $48.3^{\circ}$ and a thickness of $0.4 \mathrm{~mm}$. The rotation speed can be varied between 3100 and $28600 \mathrm{r} \mathrm{min}^{-1}$ in order to select the desired neutron wavelength (Wagner et al., 1992).
The D33 selector is mounted on a translation table, allowing it to be moved in and out of the beam path for monochromatic and TOF modes, respectively. A rotation table in the horizontal plane allows the angular orientation of the selector relative to the mean neutron beam direction to be adjusted, effectively modifying the projected angular opening between helical blades, which in turn allows the resolution (and minimum and maximum wavelengths) to be modified by between 5 and $20 \%$ for angular orientations of the selector of $\pm 8^{\circ}$, respectively. When the selector is moved out of the beam path it is replaced by an evacuated tube with no neutron guide. Since the insertion of the velocity selector presents the longest gap in the guide system $(40 \mathrm{~cm})$, the guides after the selector and throughout the rest of the instrument have reduced dimensions of $30 \times 30 \mathrm{~mm}$. This intended step-down in guide dimension has the effect of limiting divergence losses due to a gap in the guide system, or eliminating them completely depending on the final accepted divergence and wavelength, as can be shown by simple ray tracing or Monte Carlo simulations.

\subsection{Chopper system}

The TOF method offers several advantages for SANS and is the optimum method for instruments at pulsed spallation neutron sources (Ishikawa et al., 1986; Seeger \& Hjelm, 1991; Heenan et al., 1997, 2006, 2011). A pulsed broad wavelength band, for example between 2 and $20 \AA$, increases the dynamic $q$ range for one instrument configuration by a factor of 10 compared with a monochromatic measurement. Furthermore, a TOF SANS instrument can also offer much higher and more flexible resolution possibilities than are available with a

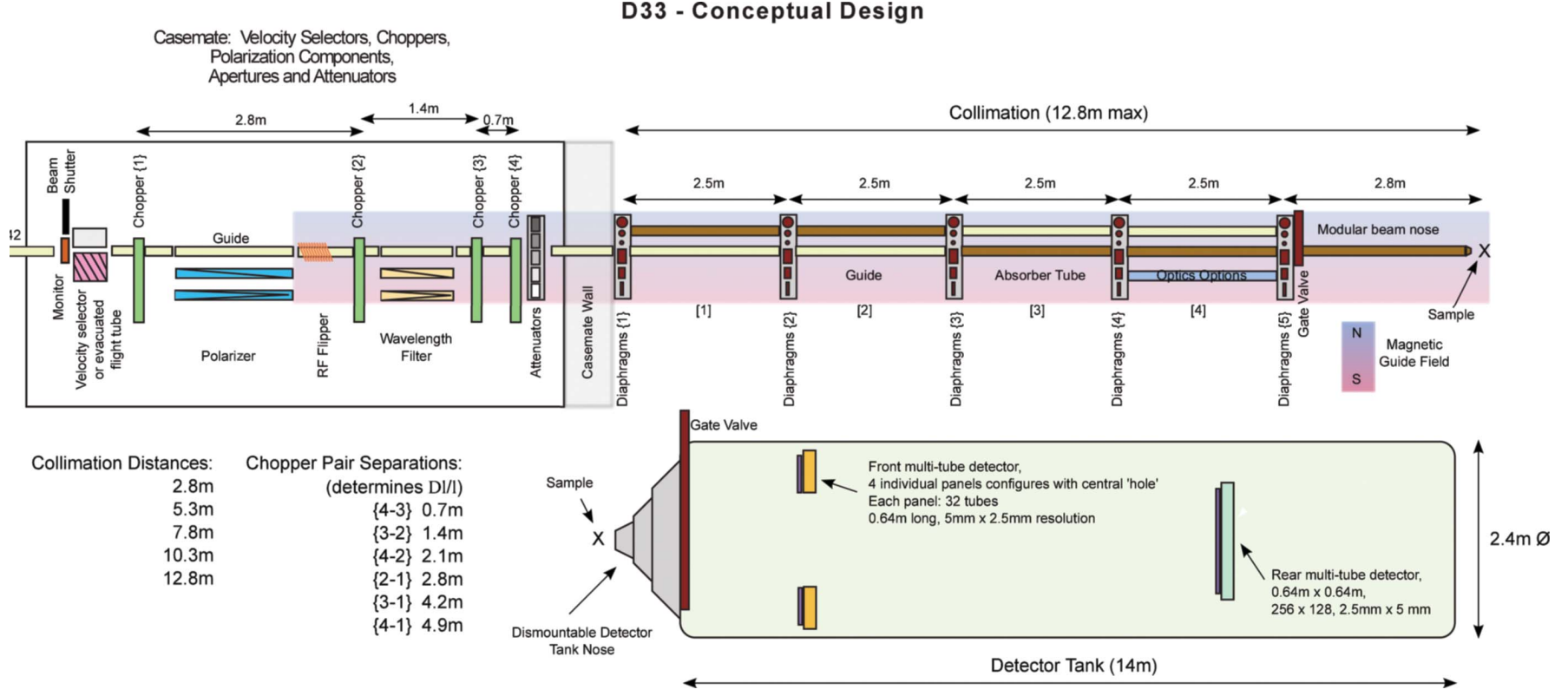

Figure 1

A schematic drawing of the D33 SANS instrument. The enclosed 'casemate' section contains optical beam components such as the velocity selector, choppers, polarizers, wavelength filters and attenuators. A maximum collimation distance of $12.8 \mathrm{~m}$ and a system of inter-collimation section diaphragms define the angular divergence of the neutron beam on the sample. A $14 \mathrm{~m}$ long $\times 2.4 \mathrm{~m}$ diameter detector tank houses two movable detector trolleys accommodating the five panels of multi-detectors. 
velocity selector and allows access to short neutron wavelengths.

D33 uses the double-chopper system (van Well, 1992) already used on the D17 and Figaro (ILL) (Cubitt \& Fragneto, 2002; Campbell et al., 2011), Platypus (ANSTO) (James et al., 2006) and REFSANS (Munich) (Kampmann et al., 2006) reflectometers. When operated in the 'optically blind' configuration, the double-chopper system has the key advantage that the wavelength resolution, $\Delta \lambda / \lambda$, is independent of the wavelength, $\lambda$. This should be compared with the case of a single disc chopper where $\Delta \lambda / \lambda$ decreases as $1 / \lambda$, which is far from ideal for a TOF SANS instrument. In the zero-phase optically blind configuration, a large window in the first upstream chopper allows neutrons to pass and continuously fill the space, $\Delta D$, up to the second chopper. The large window of the second chopper opens at the same time as the window on the first chopper closes. The neutron pulse containing all wavelengths is therefore shaped by the simultaneous opening and closing of the second (leading edge of the pulse) and first choppers (tail end of the pulse), respectively. The uncertainty in the starting position of the pulse when the chopper opens is therefore the spacing $\Delta D$ between the chopper pair, relative to the total flight path from the mid-point of the chopper pair to the detector, $D$. For a particular neutron wavelength (velocity) this corresponds to an uncertainty in time, $\Delta t$, relative to the total flight time to the detector, $t$. Since neutron wavelength is inversely proportional to velocity,

$$
\Delta \lambda / \lambda=\Delta t / t=\Delta D / D
$$

hence the fractional wavelength resolution is constant for all wavelengths and depends only on the ratio of the chopper spacing, $\Delta D$, and the final flight path to the detector, $D$. Since $\Delta t(\lambda)$ defines the effective opening time of the doublechopper system, the transmission $T$ of the choppers can be written as

$$
T(\lambda)=\Delta t(\lambda) f
$$

where $f$ is the chopper frequency. The transmission of the double chopper therefore increases linearly with wavelength. This is fortuitous and goes some way to undoing the rapidly decreasing intensity of the neutron spectrum at long wavelengths $\left(\sim 1 / \lambda^{5}\right)$. The maximum pulse repeat frequency, $f_{\max }$, to avoid frame overlap is determined by the time taken for the longest wavelength, $\lambda_{\max }$, to reach the detector. Hence

$$
f_{\max }=\frac{v_{\min }}{D}=\frac{h}{\left(D m_{\mathrm{n}} \lambda_{\max }\right)} \quad(\mathrm{Hz}),
$$

where $v_{\min }$ is the neutron velocity at wavelength $\lambda_{\max }, h$ is the Planck constant and $m_{\mathrm{n}}$ is the neutron mass. The maximum chopper frequency is therefore inversely proportional to the longest useful wavelength $\lambda_{\max }$ and $D$. Finally, the window opening, $\alpha$, in the individual chopper discs should be sufficiently large to allow the longest wavelength $\lambda_{\max }$ to traverse the chopper pair separation $\Delta D$ and fill or exit the chopper system, hence

$$
\alpha=360 \Delta \lambda / \lambda \quad \text { (degrees). }
$$

For example, for the lowest wavelength resolution on D33 of $25 \%$, the chopper windows should have an opening of at least $90^{\circ}$. D33's choppers have openings of $110^{\circ}$, as discussed below.

Fig. 1 shows that the chopper system consists of four independent choppers with separations of $2.8,1.4$ and $0.7 \mathrm{~m}$, where the last chopper $\{4\}$ is at distance of $14.1 \mathrm{~m}$ from the nominal sample position. Four choppers allow six possible chopper pair spacings and wavelength resolutions for a particular detector distance. The chopper separations have been determined so as to allow $\Delta \lambda / \lambda$ of approximately $10 \%$ for all possible sampledetector distances, while also providing the option of both higher and lower resolutions between 2.6 and $25 \%$.

Fig. 2 shows a comparison of the measured neutron flux on D33 as a function of $\Delta \lambda / \lambda$ for collimation and detector distances of $12.8 \mathrm{~m}$. Early simulations showed that the flux of D33 in TOF mode with a wavelength band between 2 and $20 \AA$ and $\Delta \lambda / \lambda=10 \%$ should be approximately equivalent to that of a velocity selector running at $8 \AA$ and of the same resolution (Dewhurst, 2008). Fig. 2 shows that this is indeed the case. Fig. 2 also shows that TOF using a narrower band between 2 and $12 \AA$ uses the more intense part of the spectrum and has an approximately $25 \%$ higher flux than a broader band up to $20 \AA$ A. In addition, Fig. 2 shows that the flux in TOF mode increases linearly with decreasing resolution, as expected from the properties of the optically blind double chopper [equations (1) and (2)].

Equation (1) overlooks the effect that a finite-sized neutron guide has on the uncertainty $\Delta t$ in the time origin of the pulse. The long flight path and broad wavelength band mean that typical chopper frequencies are low and of the order of 5$15 \mathrm{~Hz}$. The time it takes the 'slowly' rotating chopper to open or close over the cross section of the neutron guide leads to an additional timing uncertainty, $\Delta t_{\mathrm{g}}$. This, in turn, leads to limitations in the highest wavelength resolutions that can be achieved at short wavelengths. The original design of D33

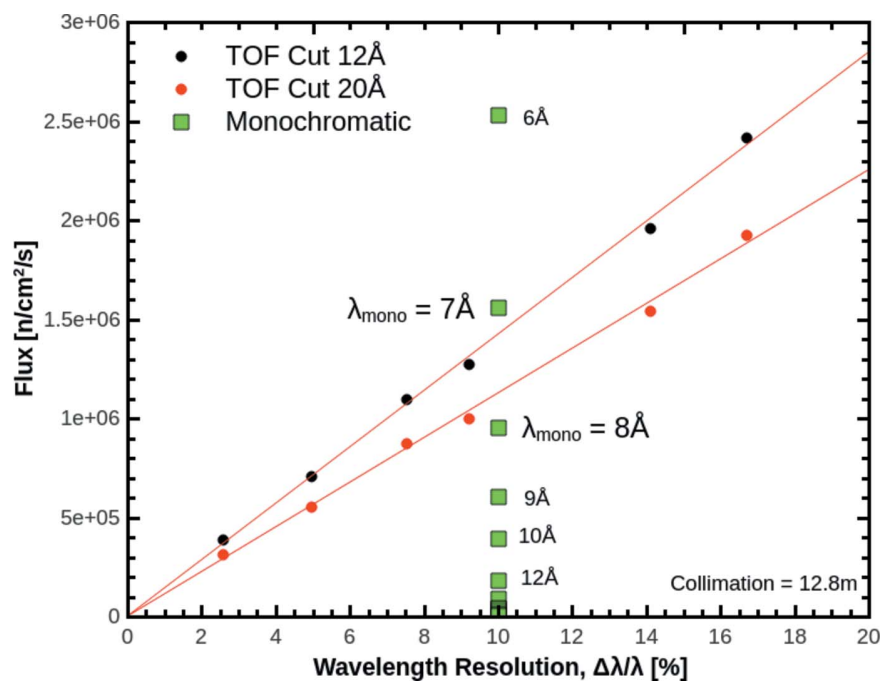

Figure 2

Neutron flux at the sample position on D33 as a function of wavelength resolution $\Delta \lambda / \lambda$, in both monochromatic (green squares) and TOF modes with wavelength cut-offs at 12 (black circles) and $20 \AA$ (red circles). In TOF mode the neutron flux increases linearly with decreasing resolution. 


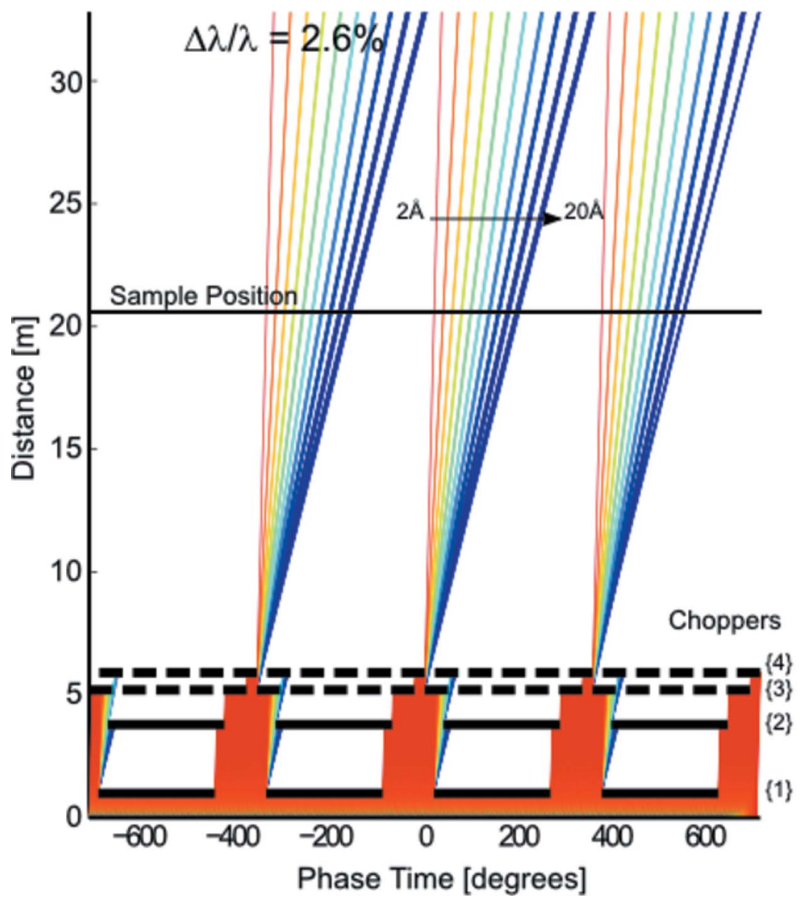

(a)

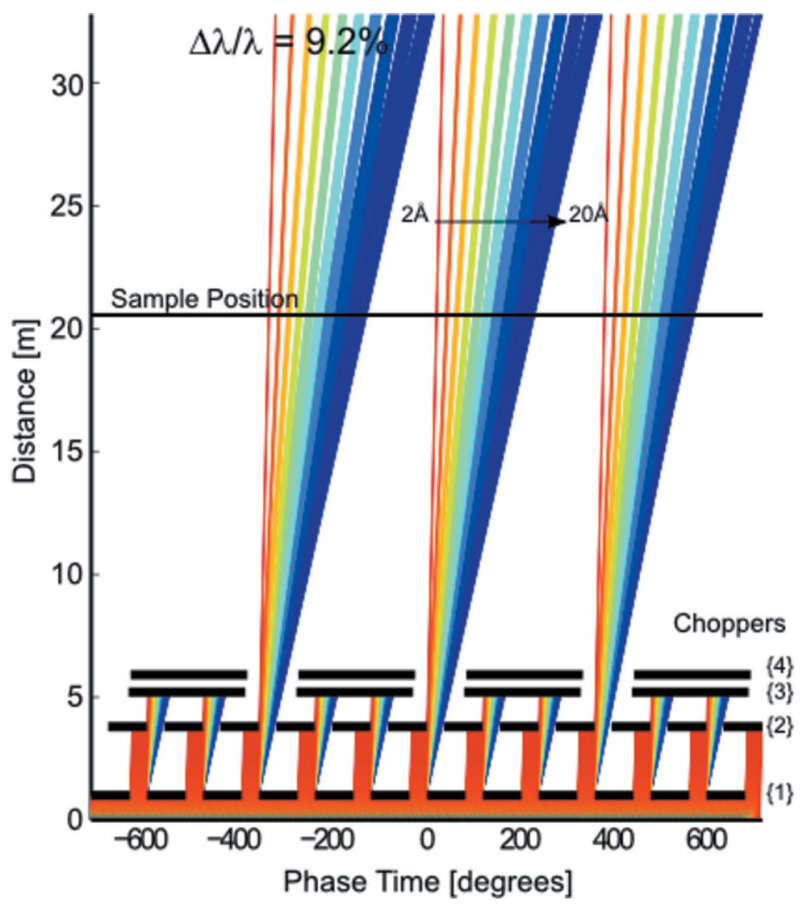

(b)

Figure 3

Time-distance diagrams for the D33 chopper system for resolutions of $(a) \Delta \lambda / \lambda=2.6 \%$ and $(b) \Delta \lambda / \lambda=9.2 \%$, with the rear detector $12.8 \mathrm{~m}$ from the sample position. In part $(a)$ the master double-blind chopper pair $\{3\}$ and $\{4\}$ cut the neutron beam at $N=5$ times the nominal TOF frequency, $f_{\text {max }}$, while choppers $\{1\}$ and $\{2\}$ perform a pulse pre-selection. In part $(b)$ choppers $\{1\}$ and $\{2\}$ cut the neutron beam at $N=3$ times $f_{\max }$, while choppers $\{3\}$ and $\{4\}$ perform a pulse selection of one in every three pulses.

(Dewhurst, 2008) described a neutron guide cross section of $50 \times 50 \mathrm{~mm}$ and a $20+20 \mathrm{~m}$ instrument. The large guide cross section and slow chopper rotation speed would have limited the highest resolution to $14 \%$ for the shortest wavelengths. A reduction in $\Delta t_{\mathrm{g}}$ of approximately a factor 10 was required in order to achieve $\sim 2.6 \%$ resolution over the entire wavelength band. A re-evaluation of the maximum sample size of $15 \mathrm{~mm}$ allowed a re-dimensioning of D33 by a factor of $\sim 2 / 3$ to a $12.8+12.8 \mathrm{~m}$ instrument with a reduced guide size of $30 \times$ $30 \mathrm{~mm}$. A shorter instrument allows higher chopper frequencies $f_{\max }$ which, combined with a reduced guide size, reduces the guide cutting time $\Delta t_{\mathrm{g}}$.

A second and rather elegant solution involves more optimally using the four-chopper system already in place. At any one time only two of the four choppers are required to define the pulse. By rotating these 'master' resolution-defining choppers at multiples of the nominal frequency, $N f_{\max }$, multiple neutron pulses can be produced which would normally result in frame overlap at the detector. The two 'spare' choppers rotating at $f_{\max }$ then can be used to perform a frame selection task, allowing only one in $N$ pulses to the sample and detector. An increased chopper speed necessarily requires an increased opening angle $\alpha$, also by a factor $N$. The minimum of these two criteria determines the maximum master chopper speed multiplication $N$ that can be used for a particular configuration. The first is given by the available opening angle $\alpha$ of the chopper windows. The second is that frame overlap must not occur between the separation of the master chopper pair. The result of both these criteria is, for example, that to achieve the highest resolution of $2.6 \%$, at the furthest detector distance of $12.8 \mathrm{~m}$, the master choppers can rotate at up to $N=5$ times the nominal frequency $f_{\max }$. This, combined with the reduced guide size and shorter instrument length, reduces $\Delta t_{\mathrm{g}}$ by more than one order of magnitude. At the lowest resolution of $25 \%$, with the detector at $2 \mathrm{~m}$, no speed multiplication is required or indeed possible $(N=1)$ because of limitations in the chopper opening angle.

The overall effect of the master chopper speed multiplication is to reduce the chopper-guide cutting time $\Delta t_{\mathrm{g}}$ by a factor $N$ while all other properties of the double-chopper system remain unchanged. Fig. 3 shows two examples of timedistance diagrams that show pictorially how the master chopper speed multiplication works in the context of the fourchopper system. Fig. 3(a) shows the highest-resolution example of $\Delta \lambda / \lambda=2.6 \%$, where the master pair choppers $\{3\}$ and $\{4\}$ turn at $5 f_{\max }$. The 'spare' choppers $\{1\}$ and $\{2\}$ make a frame pre-selection turning at $f_{\max }$, thus preventing too many pulses and frame overlap at the detector. Fig. 3(b) shows a second example with $\Delta \lambda / \lambda=9.2 \%$, where the master pair choppers $\{1\}$ and $\{2\}$ turn at $3 f_{\max }$, while choppers $\{3\}$ and $\{4\}$ perform a pulse selection of one in every three.

\subsection{Wavelength filters}

The TOF mode described above assumes a maximum wavelength cut-off $\lambda_{\max }$ above which no neutrons are present and determines the maximum frequency of the choppers $f_{\max }$ to avoid frame overlap at the detector. D33 allows one of three 
available wavelength bands to be chosen by the insertion of one of two wavelength cut-off filters (between choppers $\{3\}$ and $\{4\})$, or no filter and a natural wavelength cut-off given by the intrinsic spectrum from the cold source. The wavelength cut-off filters consist of a $0.84 \mathrm{~m}$ section of neutron guide with a reflecting mirror at a shallow angle to the guide. The mirror is set at an angle, $\theta_{\mathrm{m}}$, such that long-wavelength neutrons, $\lambda>$ $\lambda_{\max }$, are reflected by the mirror and absorbed by the surrounding guide cavity. Neutrons with $\lambda<\lambda_{\max }$ pass undeflected through the device with some small losses due to absorption in the silicon mirror substrate. For an efficient long-wavelength rejection, the mirrors should have an extremely high reflectivity at the critical edge, greater than 0.99 , and be coated on both sides of the silicon mirror substrate. A second constraint on the mirror angle and mirror and guide coatings requires that the unwanted reflected neutrons are absorbed by the surrounding neutron guide wall and are not

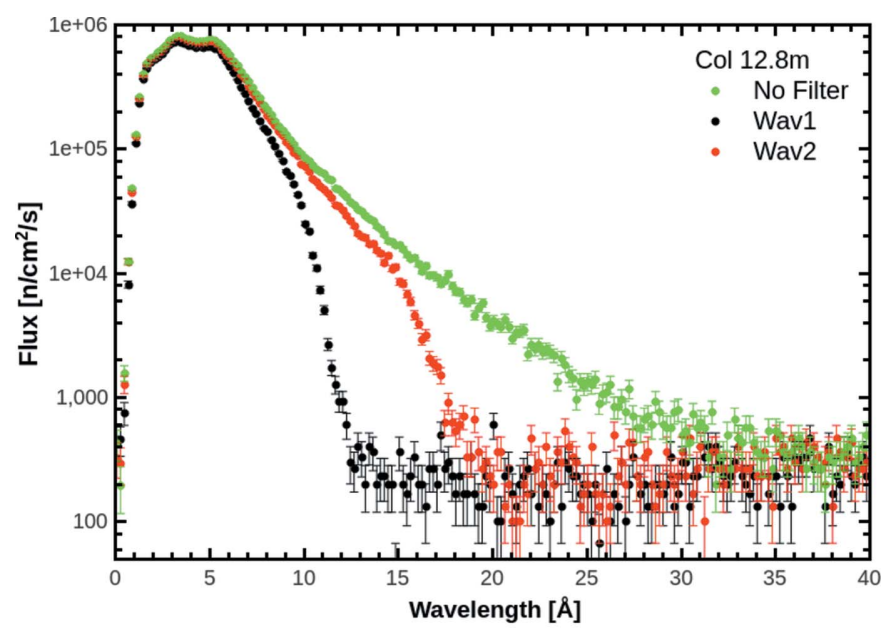

(a)

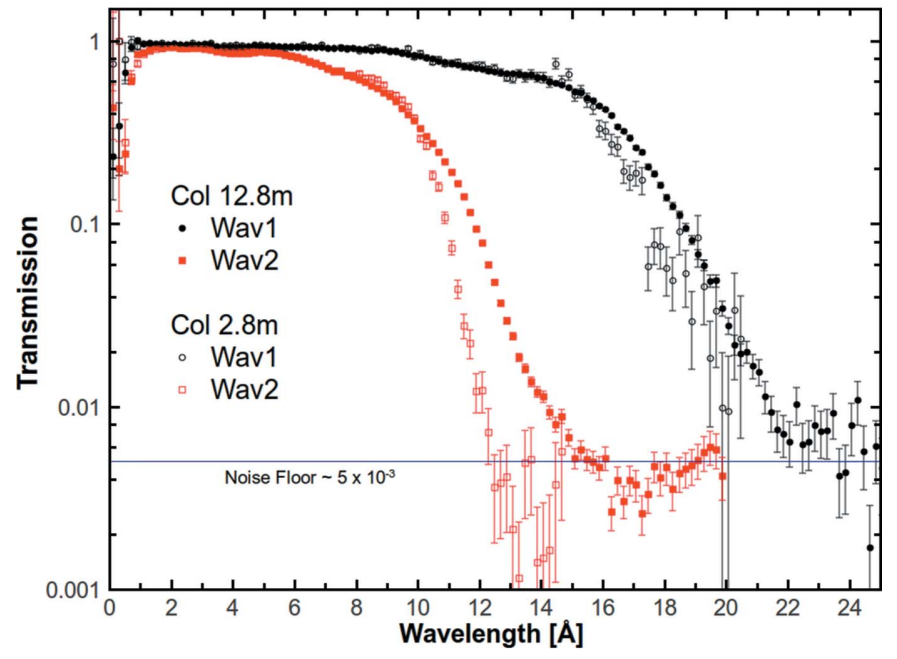

(b)

Figure 4

(a) The flux at the sample position on D33 as a function of wavelength, measured in TOF mode $\left(\lambda_{\max }=40 \AA\right.$ ) with a collimation of $12.8 \mathrm{~m}$ for no wavelength filter (green), a $20 \AA$ cut-off mirror (Wav 1, black) and a $12 \AA$ cut-off mirror (Wav 2, red). (b) The transmission of Wav 1 (black) and Wav 2 (red) as a function of wavelength and beam divergence for collimation distances of $2.8 \mathrm{~m}$ (open symbols) and $12.8 \mathrm{~m}$ (solid symbols). reflected by the guide back towards the mirror at higher angles. The subsequent criteria for an effective wavelength cut-off filter to be used in transmission become rather complex when considering the incoming divergence of the neutron beam and the divergence that is finally accepted by the instrument. Briefly, in order to avoid unwanted long-wavelength contamination for all divergence, $m$ for the reflective coating of the mirror, $m_{\text {mirror }}$, should be more than twice that of the surrounding neutron guide wall, $m_{\text {guide }}$ (Dewhurst, 2012).

Two wavelength cut-off filters are available on D33, with nominal cut-offs of 20 and $12 \AA$. The $20 \AA$ cut-off mirror consists of a single blade (made from many pieces) of $m_{\text {mirror }}=$ 1.1 multilayers of alternating nickel-vanadium and titanium layers coated onto both sides of a $0.5 \mathrm{~mm}$ thick silicon substrate. The mirror is mounted at an angle of $2.2^{\circ}$ inclined in the vertical direction in a groove machined in the walls of the surrounding $0.84 \mathrm{~m}$ long neutron guide. The $12 \AA$ cut-off mirror consists of similar mirror pieces mounted in a $\mathrm{V}$ geometry, with a small overlap at the apex pointing in the upstream direction and at an angle of $1.32^{\circ}$. The surrounding guide is left as uncoated Borofloat glass of $m_{\text {guide }}=0.65$ for the guide surfaces opposite and parallel to the mirror. A detailed discussion of the design criteria for wavelength cut-off mirrors in neutron guides and the specification of the D33 wavelength filters is given by Dewhurst (2012). Fig. 4(a) shows the neutron flux measured for a collimation of $12.8 \mathrm{~m}$, both with and without the wavelength cut-off filters for a wavelength resolution of $\sim 10 \%$ and for a $\lambda_{\max }$ of $40 \AA$. The overall form of the spectrum is as expected for the ILL cold source and is essentially that of a Maxwellian truncated below $\sim 1.5 \AA$, owing to the up-stream curvature of the neutron guide. Two maxima in the spectrum at 3.3 and $5 \AA$ border a broad local minimum around $4.5 \AA$ due to Bragg scattering from $\mathrm{Al}$ windows in the beam path. Fig. 4(b) shows the transmission of the cut-off filters as a function of wavelength for collimation distances of 2.8 and $12.8 \mathrm{~m}$. For the longest collimation (smallest divergence) the mirrors perform closest to ideal, presenting sharp cut-offs at the intended 12 and $20 \AA$, respectively. With increased divergence the cut-off broadens as expected (Dewhurst, 2012), with the transmission descending below the noise floor of the measurement beyond $\sim 14$ and $22 \AA$, respectively. The timing of the chopper system described above must take into account the real (divergencedependent) wavelength cut-off in the determination of $\lambda_{\max }$. Transmission losses through the silicon mirror substrate are also apparent in Fig. 5(b), with transmission falling below unity even for wavelengths below the cut-off. This is more evident for the $12 \AA$ cut-off mirror (Wav2) than for the $20 \AA$ cut-off (Wav1), because of the shallower mirror angle and increased path length through the silicon substrate.

\subsection{Polarization components (polarizing mirrors and RF flipper)}

One of two polarizers can be inserted into the beam between choppers $\{1\}$ and $\{2\}$, as shown in Fig. 1 . The polar- 
izers are similar in construction to the wavelength cut-off filters described above and consist of magnetized $m=3.6 \mathrm{FeSi}$ multilayer mirrors coated on both sides of a silicon substrate (Bigault et al., 2014). The polarizing mirrors are mounted in $1.2 \mathrm{~m}$ long neutron guides, which are in turn mounted in a steel frame with $\mathrm{NdFeB}$ permanent magnets, forming a figure-ofeight shaped magnetic circuit. The magnetic circuit provides a field of approximately $50 \mathrm{mT}$ in the plane of the mirror which is magnetized to saturation. A single-blade polarizer with a mirror angle $\theta_{\mathrm{m}}=1.4^{\circ}$ and a V-mirror of angle $\theta_{\mathrm{m}}=0.72^{\circ}$ provide polarized neutrons for longer and shorter wavelengths, respectively. Initial polarization tests and visual inspection of the polarizers showed that gaps of $\sim 0.5 \mathrm{~mm}$ at the extremities where the mirrors are butted up to the edge of the neutron guide allow a leakage of unpolarized neutrons, resulting in reduced polarization from the device. Small absorbing $\mathrm{B}_{4} \mathrm{C}$ rubber blinds are now mounted at the entrance

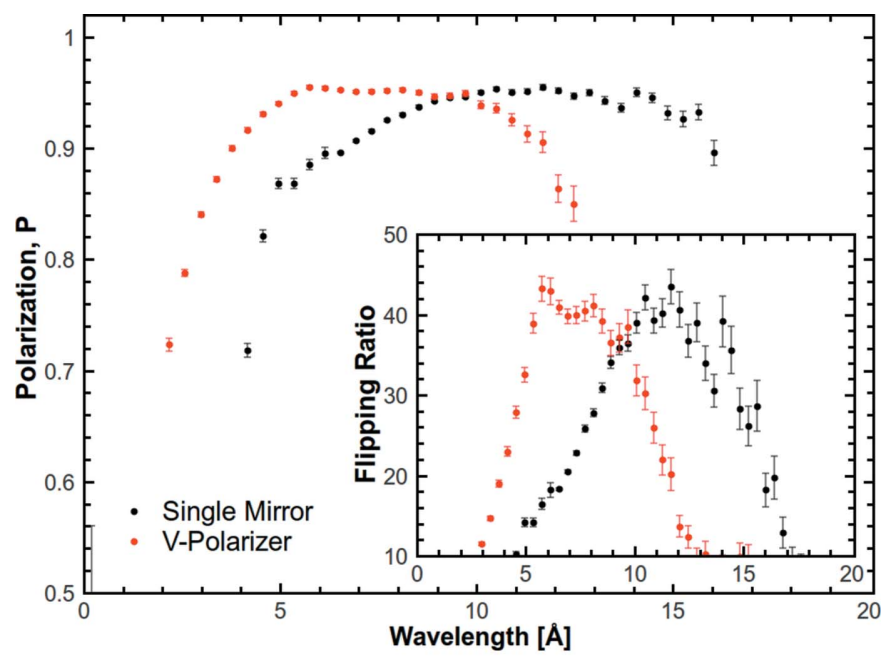

(a)

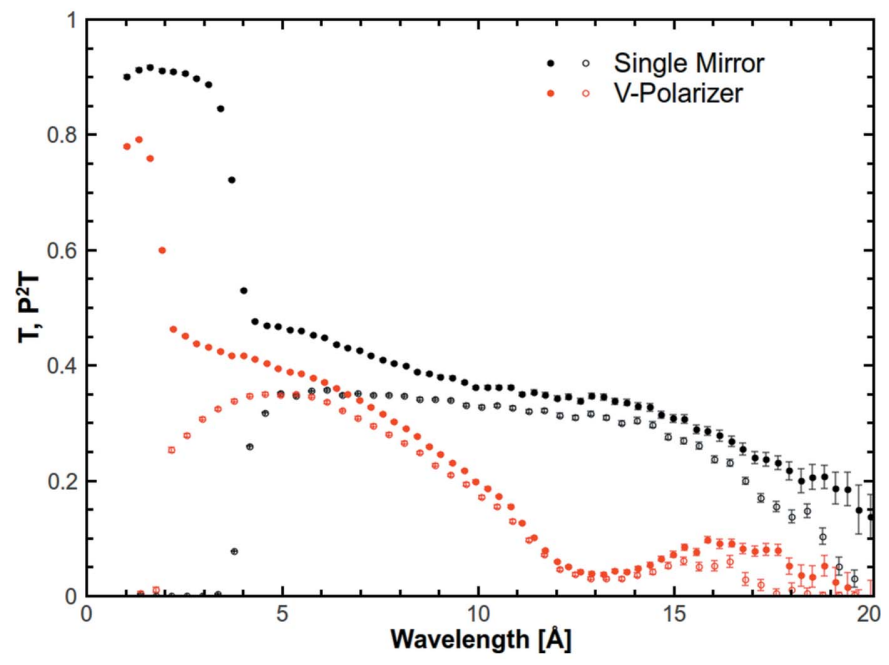

(b)

Figure 5

(a) Polarization $P$ and flipping ratio (inset) as a function of wavelength for the single-mirror (black) and V-mirror (red) polarizers. (b) Transmission of an unpolarized beam $T$ (closed symbols) and figure of merit $P^{2} T$ (open symbols) as a function of wavelength for the single-mirror (black) and Vmirror (red) polarizers. and exit of the polarizing guides to block the passage of unpolarized neutrons at the mirror edge, significantly improving the polarization with only a modest loss of neutron flux of around $5 \%$.

Fig. 5(a) shows the polarization and corresponding flipping ratio provided by the polarizers as installed on D33. As expected, at short wavelengths the polarization is low owing to the finite reflectivity of the mirrors between the total-reflection region $(m<1)$ and the supermirror critical edge at $m=$ 3.9. Below the total-reflection regime, reflection out of the beam of the unwanted spin state is expected to be close to perfect and should result in a polarization close to unity. The performance of the installed supermirror polarizer is in good agreement with the polarized neutron reflectometry characterization for the mirrors given by Bigault et al. (2014).

Fig. 5 shows the polarization $P$, transmission $T$ and figureof-merit $P^{2} T$ for both polarizers as a function of wavelength and measured for the longest collimation of $12.8 \mathrm{~m}$. The polarization was measured using a magnetized polarizing mirror in reflection and assumed to be perfectly efficient. Fig. 5(a) shows that the D33 polarizers achieve polarization $P>0.9$ for wavelengths between 7 and $18 \AA$ for the single mirror and between 3.75 and $11.5 \AA$ for the V-polarizer. There is no significant dependence of the measured polarization on the collimation length, as expected for the non-magnetic guides in the collimation. It should be noted that, while the Vpolarizer provides better polarization for short wavelengths $(\lambda$ $<\sim 8 \AA$ ), transmission losses in the silicon substrate due to the shallow angle of the mirrors are more significant than for the single-mirror polarizer. For wavelengths greater than $\sim 12 \AA$, both polarization and transmission decrease rapidly with increasing wavelength, owing to reflection by the mirror substrate of both spin states. In contrast, the single-mirror polarizer exhibits a much broader band of usable wavelengths, with high polarization and transmission giving a plateau in the $P^{2} T$ figure of merit between 5 and $15 \AA$. These characteristics make the single-mirror polarizer best suited for polarized TOF studies.

After the polarizers, an adiabatic resonance radio frequency (RF) spin flipper is installed around the polycarbonate vacuum tube housing the neutron guide and allows the neutron polarization direction to be reversed (Bazhenov et al., 1993). The spin flipper provides two orthogonal magnetic fields: a static field perpendicular to the neutron beam decreasing from 4 to $1.2 \mathrm{mT}$ along the neutron path, and an RF field produced by a solenoidal coil $(150 \mathrm{~mm}$ long, $105 \mathrm{~mm}$ diameter, 110 windings). In the flipper 'on' state the RF coil is supplied with $220 \mathrm{~mA}$ (equivalent to $B_{\mathrm{rf}} \simeq 0.2 \mathrm{mT}$ ) at an RF frequency of $51 \mathrm{kHz}$, corresponding to the neutron Larmor frequency in the central region of the spin flipper and thus causing neutron spin reversal. The flipper is limited by a theoretical minimum wavelength of $\lambda_{\min }=\pi \beta /\left(\gamma l B_{\mathrm{rf}}\right)=2 \AA$, where $\beta=3956 \AA \mathrm{m} \mathrm{s}^{-1}$ and $\gamma$ is the neutron gyromagnetic ratio (Grigoriev et al., 1997). The RF flipper efficiency was determined using the four POLARIS empty-beam transmission measurements using a polarized ${ }^{3} \mathrm{He}$ cell according to Krycka et al. (2012) and is simply the ratio between the 
polarization in the RF on state versus the polarization in RF off. The difference between the two polarizations is given by the efficiency of the RF flipper. For wavelengths longer than $2 \AA$ the RF flipper performance is better than 0.99 , e.g. 0.993 (2) at $5 \AA$ and 0.998 (6) at $6 \AA$.

In order to retain the neutron polarization along the flight path from polarizer to sample, magnetic guide fields of the order of $1 \mathrm{mT}$ have been installed, using permanent $\mathrm{NbFeB}$ magnets and steel plates to form the magnetic circuit. Polarization analysis or discrimination of the scattered spin state after sample interaction can be made by the installation of a polarized ${ }^{3} \mathrm{He}$ gas-filled silicon-faced quartz cell of length 100 or $150 \mathrm{~mm}$ and $120 \mathrm{~mm}$ diameter. The ${ }^{3} \mathrm{He}$ cell is placed in a so-called 'magic box', which provides a constant and homogeneous magnetic field over the cell in order to preserve the ${ }^{3} \mathrm{He}$ spin orientation. The magic box is also equipped with an RF coil, set to allow the ${ }^{3} \mathrm{He}$ spins to be flipped via an adiabatic RF pulse. The generous sample zone dimensions of D33 allow the ${ }^{3} \mathrm{He}$ cell and magic box to be installed in the sample zone in front of the detector tank, allowing convenient access for the regular (daily) replacement of the ${ }^{3} \mathrm{He}$ cells.

\subsection{Attenuators}

A translation device allows the insertion of one of three attenuators into the neutron beam. The attenuators are constructed as 'sieve'-type meshes, consisting of an array of $8 \times 8$ holes uniformly distributed over the $30 \times 30 \mathrm{~mm}$ beam cross section. The holes are machined in a sandwich of materials consisting of a coarse array of holes in $2 \mathrm{~mm}$ sintered ${ }^{10} \mathrm{~B}_{4} \mathrm{C}$, a fine array of beam-defining holes in gadolinium foil, and a coarse array of holes in an aluminium mechanical support. The advantage of sieve-type attenuators is that they reduce the outgoing beam intensity by a factor close to their geometric area sampling of the beam. Their attenuation is in principle independent of both wavelength and collimation (divergence), although finite spatial sampling of the beam and divergence can lead to some subtle dependence of the attenuation on collimation length, but they are essentially independent of wavelength. This should be compared with absorption-type attenuators that can uniformly sample the entire beam cross section but have an exponentially increasing attenuation as a function of wavelength and are far from ideal for a TOF instrument. Attenuators 1 to 3 comprise beamdefining holes of $0.4,0.2$ and $0.1 \mathrm{~mm}$ diameter with nominal geometric attenuations of 112, 447 and 1790, respectively. The three attenuators on D33 have been calibrated to give values for the weakest attenuator (No. 1) between 110 and 135 for collimation lengths of 2.8 and $12.8 \mathrm{~m}$, respectively. Attenuators Nos. 2 and 3 behave similarly, with a fixed ratio between attenuators 2 and 1 of 3.89 ( $c f$. a geometric ratio of 4$)$ and one between attenuators 3 and 1 of 17.12 ( $c f$. a geometric ratio of 16).

2.8. Collimation section, source definition apertures and beam input nose

The collimation section of D33 defines the angular divergence of the neutron beam onto the sample. Collimation lengths of 2.8, 5.3, 7.8, 10.3 and $12.8 \mathrm{~m}$ can be selected with the aid of four $2.5 \mathrm{~m}$ long changer sections (numbered [1] to [4] in Fig. 1), allowing either the positioning of a neutron guide or an empty flight path consisting of an absorbing boron carbidelined tube and antiparasitic baffles. Five diaphragm changer translation stages (diaphragms $\{1\}$ to $\{5\}$ in Fig. 1) allow the positioning of one of six apertures in the neutron beam path at the entrance and between sections of the collimation. The diaphragms are machined identically in sintered strips of ${ }^{10} \mathrm{~B}_{4} \mathrm{C}$ and have circular openings of $5,10,20$ and $30 \mathrm{~mm}$ in diameter and rectangular shapes of $32 \times 32 \mathrm{~mm}$ (i.e. just larger than the guide size) and 32 (horizontal) $\times 45 \mathrm{~mm}$ (vertical), off-centred vertically in order to allow for a parabolic flight path due to gravity when using the longest collimation distances and wavelengths (Cubitt et al., 2011). Under normal operation, only the diaphragm set between the last piece of guide and the first absorbing tube section is of any practical use in defining the source aperture size. The combination of collimation lengths and source apertures allows the angular divergence of the beam to be defined between \pm 0.01 and $\pm 0.31^{\circ}$. Indeed, D33 has some engineering redundancy in the placement of the diaphragm sets at the intersection of every collimation block, but this allows flexibility in the instrument configuration and the development of novel scattering methods such as those using multiple beams (Dewhurst, 2014). The final $2.8 \mathrm{~m}$, defining the shortest collimation distance, consist of a number of precision-made DN100 vacuum tubes in section lengths between 1 and $0.25 \mathrm{~m}$. Antiparasitic baffles occupy the O-ring joint supports between adjacent tube sections, and a final beam nose of reduced diameter supports a single-crystal sapphire window and beam-defining sample apertures. The modular nature of the final $2.8 \mathrm{~m}$ allows the minimum collimation distance to be reduced to $0.5 \mathrm{~m}$ to accommodate large sample environments, as described below.

\subsection{Sample zone}

The sample zone of D33 consists of the usual movement stages, allowing sample positioning in the vertical and two horizontal directions and rotation in the horizontal plane, and a goniometer for tilts in the vertical plane. The sample movement stages are constructed from as few magnetic components as possible and are dimensioned to carry weights up to $500 \mathrm{~kg}$. The entire sample table is mounted on top of an additional heavy-duty movement stage embedded in the floor, enabling translation of the entire sample table and sample environments along the $2.8 \mathrm{~m}$ length of the modular beam nose. As such, D33 can accommodate bulky and massive sample environments such as large cryomagnets, like that of the $17 \mathrm{~T}$ cryomagnet belonging to the University of Birmingham (Holmes et al., 2012), and allows space for positioning the ${ }^{3} \mathrm{He}$ spin analysis magic box and cell, well separated from any magnetic sample environments. The usual array of standard sample environments, common to the ILL's SANS and reflectivity instruments, can be mounted easily on D33. For materials science and physics studies these are most commonly sample environments such as electromagnet, 
cryostat, cryomagnet and furnace. For soft-matter and biology studies the standard sample environment is often an automated sample changer with up to 22 positions for rectangular optical cells with a thickness of 1 to $5 \mathrm{~mm}$. The temperature of the sample changer can be varied between 278 and $353 \mathrm{~K}$ with a double circulating bath system controlled by a solenoid valve that allows the user to switch rapidly from one temperature to another. Ancillary equipment includes items such as a rheometer, a shear cell, a stopped-flow device, an acoustic levitator and the usual array of standard sample environments. All the sample environments are remotely controlled through the instrument software Nomad.

\subsection{Detector tank and detectors}

A $2.4 \mathrm{~m}$ diameter and $14 \mathrm{~m}$ long evacuated aluminium detector tank houses two movable detector trolleys upon which are mounted the front and rear neutron detectors. The two trolleys share a common rail system, allowing the positioning of the detectors at distances of between 1.2 and $12 \mathrm{~m}$ from the sample for the front detector and 2 to $12.8 \mathrm{~m}$ for the rear detector, with a closest approach of $0.8 \mathrm{~m}$ between the two detector trolleys. All detectors are of the monoblock design, where individual ${ }^{3} \mathrm{He}$ gas tubes have been machined into a block of aluminium by spark erosion to form an array of rectangular tubes of dimensions 4.5 (width) $\times 10 \mathrm{~mm}$ (depth), with a $0.5 \mathrm{~mm}$ wall thickness between neighbouring tubes. In order to obtain tubes of $64 \mathrm{~cm}$ in length, three precisely machined blocks are welded together to form the full length. The single rear detector consists of a $64 \times 64 \mathrm{~cm}$ array $(256 \times$ 128 pixels) of 128 horizontal position-sensitive detector tubes, with a vertical resolution of $5 \mathrm{~mm}$ determined by the tube spacing and horizontal event positioning provided by a charge division of the neutron event on the resistive detection wire. The position along the wire is digitized into 256 channels, corresponding to a pixel size of $2.5 \mathrm{~mm}$ but with an intrinsic resolution along the wire length estimated to be approximately $5 \mathrm{~mm}$, matching that of the tube spacing in the vertical direction. The front detector consists of four separate monoblock panels of $64 \times 16 \mathrm{~cm}(256 \times 16$ pixels $)$ mounted horizontally and vertically. The four panels form a frame-like detector of variable central opening to suit the desired scattering angles of interest around the rear detector. The variable opening of the front panel detectors is such that they can be always be configured so as not to shadow the rear detector.

The positioning of the two detector trolleys and the configuration of the central aperture in the front detector depend on the requirements of the particular experiment and on the monochromatic or TOF mode of operation of the instrument. In monochromatic mode, the front and rear detectors are usually configured at closest approach with the central aperture of the front detector fully open. The front panels then flank the sides of the rear detector, providing almost continuous solid-angle coverage between the front and rear detectors. In such a configuration D33 provides a dynamic $q$ range of $\sim 25$ and usually requires measurements to be made at two collimation/detector positions to cover the full scat- tering curve. In TOF mode, continuous solid-angle coverage is not necessary as the broad wavelength band allows continuous coverage in $q$. Positioning of the front and rear detectors is therefore a trade-off between maximum dynamic $q$ range and counting statistics at a given $q$. For example, a detector arrangement of closest approach as for the monochromatic mode and with a TOF wavelength band of 2-20 A provides a dynamic $q$ range of $\sim 250$. On the other hand, separating the front and rear detectors to their maximum at 1.2 and $12.8 \mathrm{~m}$ from the sample allows a very large dynamic $q$ range of $>1000$.

The gas tubes themselves are filled with a mixture of 12 bar ${ }^{3} \mathrm{He}, 4$ bar Ar and 0.1 bar $\mathrm{CO}_{2}(1 \mathrm{bar}=100 \mathrm{kPa})$. The high ${ }^{3} \mathrm{He}$ pressure and $10 \mathrm{~mm}$ depth of the tubes means that an individual tube is close to $100 \%$ efficient in the detection of cold neutrons. We have measured the average efficiency of the monoblock panel array of tubes to be greater than $90 \%$ for a neutron wavelength of $6 \AA$. Since the gap of $0.5 \mathrm{~mm}$ between tubes and the pitch spacing of $5 \mathrm{~mm}$ correspond to $10 \%$ of the detector array area, the result is consistent with the assumption that the tubes themselves are close to $100 \%$ efficient. A motorized beam stop and beam-stop changer allow the positioning of one of several sintered $2 \mathrm{~mm}^{10} \mathrm{~B}_{4} \mathrm{C}$ beam stops to block the direct beam during scattering measurements and prevent damage to the detector.

\section{Instrument calibration procedures}

This section describes the various calibration procedures that allow the accurate determination of the scattering vector magnitude $q$ from the neutron wavelength, distances and scattering angles, and the absolute intensity and cross sections for the scattered neutrons as corrected for the various geometric distortions.

\subsection{Detector and TOF distance}

Sample-to-detector distances are defined and measured relative to a nominal sample 'zero position'. The choice of the zero reference position is arbitrary but is within millimetres of the instrument engineering designs of the nominal sample position. Here, the encoder positions for the two motor translation stages along the beam direction are defined as zero. The detector distances are then measured using a laser distance measurer (accuracy $\sim 1 \mathrm{~mm}$ ) from the zero position to the front face of the detector panels and the $5 \mathrm{~mm}$ thickness of the detector window is added to the measured value. An uncertainty in the detection position of recorded neutron events comes from the detector tube gas thickness $(10 \mathrm{~mm})$ and the wavelength-dependent and statistical mean detection position within the gas detection tube. The overall precision in the sample-to-detector position is therefore of the order of $5 \mathrm{~mm}$ in $10 \mathrm{~m}$ and is dominated by the detection position uncertainty within the detector itself. The TOF mode requires accurate knowledge of the total flight path distances from chopper(s) to detector(s). Using a combination of laser distance measurer and rulers, the distances between the nominal sample zero position and the centre(s) of the chopper 
disc housing(s) can be measured. The estimated precision in this procedure is $\sim 5 \mathrm{~mm}$ in $10 \mathrm{~m}$.

\subsection{Wavelength calibration}

3.2.1. Velocity selector calibration. To calibrate the velocity selector, a small beam chopper can be installed at the sample position. Accuracy in the positioning and in the delay angle of the trigger pickup are not important using the following procedure. The chopper is rotated at a frequency sufficiently slow as to allow neutrons of a given wavelength to reach the furthest detector position while avoiding frame overlap. The TOF profile of the monochromatic beam at two different detector positions, ideally separated by many metres, is measured. The TOF profile of the beam from a velocity selector is ideally triangular, because of the finite selector resolution, but may become non-triangular owing to convolution with the beam divergence, the finite beam size, the finite chopper opening and the underlying intensity spectrum. From the two TOF profiles, a triangular fit function or numerical centre of mass allows the time-centre of the pulsed monochromatic beam to be measured for both detector distances. Using the time difference $\Delta t$ for the difference in detector position $\Delta d$ allows the incoming wavelength to be calculated. The selector resolution $\Delta \lambda / \lambda$ may also be determined from the TOF profiles, although smearing of the TOF profiles due to divergence, the beam size and the chopper opening window should be accounted for. This procedure is repeated for many wavelengths given by the velocity selector. A plot of wavelength versus selector speed should be linear, passing through the origin, and allows the selector to be calibrated.

3.2.2. TOF wavelength calibration. The four choppers on D33 have window openings of $110^{\circ}$, with an optical position encoder with a nominal 'zero' set at the opening edge of the window. To calibrate neutron wavelengths accurately in TOF mode, it is necessary to calibrate the chopper delay angle $\phi$, i.e. the point when the optical coder zero signal arrives relative to the point when the window cuts the mid-point of the neutron guide, as well as the relative phasing of chopper pairs. With all distances and monochromatic wavelengths (velocity selector) calibrated, the chopper delay angle $\phi$ can be measured for each of choppers $\{1\}$ to $\{4\}$ in turn. For a given detector distance and monochromatic wavelength provided by the velocity selector, the TOF to the detector can be calculated and converted to a frequency. The chopper undergoing calibration is set to this speed. Since the time-resolved detector acquisition is triggered at the coder nominal zero, close to the opening edge of the chopper, the detector should see a 'rising edge' of neutrons arriving at the detector close to the nominal TOF. The neutron count on the detector as a function of time is actually a convolution of the time it takes the chopper edge to cut the neutron guide (linear slope function) convoluted with the wavelength spread (triangular function) given by the velocity selector, and results in a linear step function with 'rounded edges'. Fitting such a function to the opening edge of the intensity versus time measured on the detector allows the actual time $t^{\prime}$ between the chopper electronic trigger and arrival at the detector to be measured. The difference, $t^{\prime}-t$, is the chopper delay time, easily converted to a physical chopper delay angle $\phi$ from the chopper speed. When all chopper delay angles have been measured, the detector acquisition and chopper phasing can be adjusted accordingly.

\subsection{Detector position calibration, relative efficiency and parallax}

Position calibration along the length of the detector tubes is made using a cadmium mask with precisely machined slits, which is mounted directly on the front window of the detector panels. A 'flat' scattering sample, such as water or Plexiglas, allows uniform illumination over the entire detector surface with neutrons only detected at the positions of the slits. The 'raw' pixel positions of the slits are then determined by a centre-of-mass calculation. A plot of raw pixel position versus physical position shows the position sensitivity of the tubes to be highly linear but with different offsets and gradients for each tube. These parameters are then used to recalibrate the position in the data acquisition software. Similarly, the 'flat' sample image allows the measurement of the individual relative pixel efficiencies with a typical r.m.s. variation in efficiency of about $5 \%$. Placing the detector sufficiently far from the sample, e.g. $8 \mathrm{~m}$, and using a $3 \mathrm{~cm}$ thick piece of Plexiglas to scatter the incoming neutron beam uniformly over $4 \pi$ steradians, the beam stop can be removed and the relative efficiency of the entire detector(s) can be measured. The efficiency measurement should be made over a sufficiently long time as to provide a 'flat' image of high statistical quality. After performing geometric solid-angle corrections to take into account the response of the flat detection plane of the detector measuring a spherically uniform scatterer, subsequent division of the measured image by its mean intensity results in the detector efficiency map of values fluctuating around unity and can be used subsequently to normalize measured scattering patterns in the data analysis software.

Parallax distortions in the measured scattering data arise primarily as a result of the $0.5 \mathrm{~mm} \mathrm{Al}$ walls between adjacent detector tubes, with a small contribution due to the finite depth of neutron detection within the position-sensitive gas tubes. For short detector distances, neutrons incident on the $0.5 \mathrm{~mm}$ border regions between tubes have the possibility to traverse the $0.5 \mathrm{~mm}$ tube side-wall and be detected in a neighbouring tube. As such, parallax distortions become more severe as the detector moves closer to the sample. Again, using a uniform spherical scatterer such as Plexiglas allows the measurement of this effect and the incorporation of corrections into the data treatment software. We find empirically that the effective count rate for a given detector tube increases approximately linearly with increased scattering angle perpendicular to the tube direction. In other words, on D33 the parallax correction is essentially one dimensional and is approximately $5 \%$ for a scattering angle of $9^{\circ}$.

\subsection{Absolute intensity}

The absolute scaling of scattering data is achieved after background subtraction and geometric and efficiency correc- 
tions, and by normalizing the scattering data to the incoming direct-beam intensity. In order to prevent damage to the neutron detectors and to remain at count rates subject to only small dead-time corrections, the direct beam can be measured on the rear or front panel detectors using the calibrated attenuators as discussed in \$2.7. The accuracy of the absolutescale calibration is then essentially that given by the calibration of the attenuation factors as a function of collimation and wavelength. The software GRASP (http://www.ill.fr/lss/grasp) includes tabulated values of the attenuation coefficients for all D33 attenuators as a function of collimation and wavelength.

\section{Instrument performance}

D33 received its first neutrons in June 2012, which saw the beginning of live instrument commissioning and delivery of neutrons to the instrument through the newly rebuilt H14 guide. Of primary concern was the neutron flux delivered to the instrument. Gold foil activation measurements at the exit of the H14(2) guide at the beginning of the D33 casemate showed the neutron flux to be $1.2 \times 10^{10} \mathrm{n} \mathrm{cm}^{-2} \mathrm{~s}^{-1}$ for the white neutron beam integrated over the available divergence, and this is in agreement with simulations of the reactor, vertical cold source and H14 guide. The inset to Fig. 6 shows the neutron flux measured on the D33 rear detector at a wavelength of $6 \AA$ and as a function of collimation length, while the main panel shows the neutron flux for D11, D22 and D33 plotted as a function of the angular resolution of the beam (determined by the guide size and collimation length). The maximum absolute neutron flux of D33 is lower than that of the D22 instrument for a similar collimation length, providing a maximum of $\sim 3 \times 10^{7} \mathrm{n} \mathrm{cm}^{-2} \mathrm{~s}^{-1}$ at $6 \AA$ and for the shortest collimation length of $2.8 \mathrm{~m}$. On the other hand, it is crucial to remember that the guide cross section of D33 is reduced to $30 \times 30 \mathrm{~mm}$ compared with that of $\mathrm{D} 22$, which has a cross section of 40 (horizontal) $\times 55 \mathrm{~mm}$ (vertical). This means that neutron flux should not simply be compared at

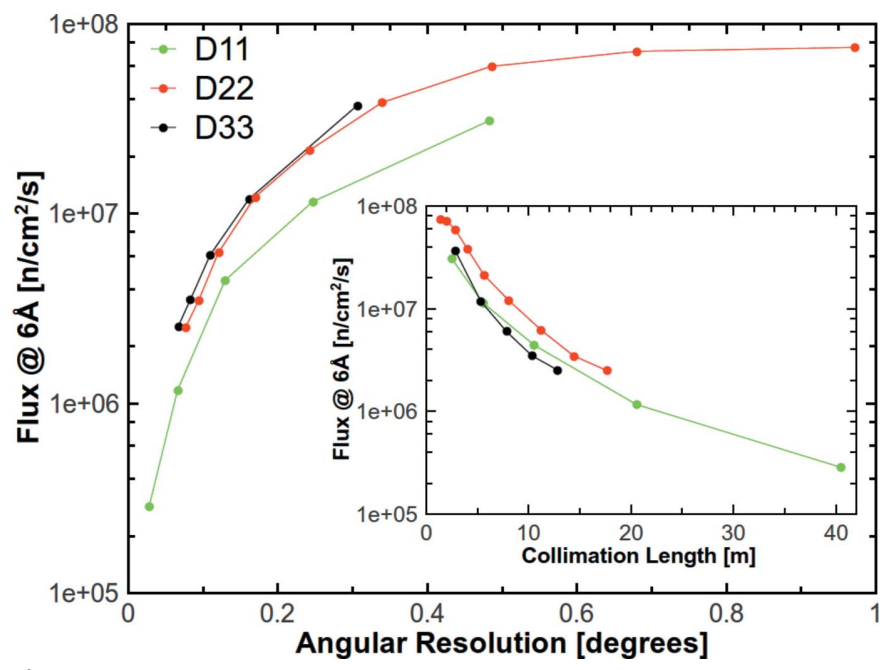

Figure 6

The flux at $6 \AA$ as a function of collimation length (inset) and of angular resolution of the beam for the ILL's SANS instruments D11, D22 and D33. equivalent collimation lengths but should be compared taking into account the angular resolution of the neutron beam $\Delta \theta$, as well as the wavelength spread $\Delta \lambda$, as shown in Fig. 6 . When compared as a function of the (mean) angular resolution of the beam, the neutron flux on D33 is in fact up to $10 \%$ higher than that of D22. In other words, a collimation distance of $2.8 \mathrm{~m}$ and guide size of $30 \times 30 \mathrm{~mm}$ on D33 delivers approximately the same flux and resolution as D22 configured with a collimation of $4 \mathrm{~m}$ for its guide dimensions of $40 \times$ $55 \mathrm{~mm}$. The fact that D22 and D33 display almost equivalent neutron fluxes for the same resolution suggests that the two cold sources in the ILL reactor, horizontal (HCS) and vertical (VCS), have approximately the same brightness. Fig. 6 also shows that the flux available at the D11 instrument is approximately half that of D22 or D33, despite being supplied with cold neutrons from the same cold source as D33. We believe this is due to the rather old $\mathrm{H} 15$ neutron guide to the instrument, dating from 1972, which presumably suffers from significant losses in reflectivty caused by aging and misalignment effects. The $\mathrm{H} 15$ guide is due to be replaced as part of the ILL's Endurance upgrade programme. It should also be pointed out that the D33 instrument lies at a distance of more than $100 \mathrm{~m}$ from the ILL reactor, thus demonstrating the efficient transport of neutrons through the rebuilt and rather long H14 guide.

Fig. 7 shows scattering data measured in monochromatic mode from a sample of silver behenate powder, $\mathrm{AgC}_{22} \mathrm{H}_{43} \mathrm{O}_{2}$. This is a silver salt of behenic acid with a relatively long layer spacing in the $c$-axis direction of $d_{001}=58.38 \AA(q=$ $0.1076 \AA^{-1}$ ) and as such it has been proposed as a calibration standard for low-angle X-ray and neutron diffraction instruments (Huang et al., 1993; Gilles et al., 1998). Fig. 7(a) shows the two-dimensional scattering pattern recorded on the D33 multi-detectors at a wavelength of $10 \AA(\Delta \lambda=10 \%)$, a collimation length of $5.3 \mathrm{~m}$, and with the rear detector at $2 \mathrm{~m}$ and the front panels at $1.2 \mathrm{~m}$ (top and bottom) and $1.39 \mathrm{~m}$ (left and right) from the sample. The front panel detectors are also opened to their maximum of $400 \mathrm{~mm}$, such that, in a flat projection, their inner edges just border the edges of the rear detector. Standard corrections were applied to the data from the rear and front detectors, as described in $\$ 3$, including the removal of background scattering and corrections for sample transmission, solid angle and parallax, and normalization to the incoming beam intensity measured on the rear detector. The rear detector captures the first-order Bragg powder diffraction peak, while the second-order peaks fall on the surrounding panels of the front detector. Fig. 7(b) shows the azimuthal regrouping of the two-dimensional data, where contributions from the rear detector, and from pairs of horizontal and vertical panels of the front detector, are plotted separately. The data from the different detectors are presented seperately in Fig. 7(b) so as to show clearly their individual contributions to the overall scattering curve. The overall $q$ range covered by this configuration extends from $0.011 \AA^{-1}$ close to the beam stop on the rear detector to $0.282 \AA^{-1}$ (dynamic $q$ range of 25), with data from the rear detector terminating at a maximum $q$ of $0.138 \AA^{-1}$. The panels of the 
front detector effectively double the dynamic $q$ range of the rear detector alone.

Fig. 8 shows scattering data measured in both TOF and monochromatic modes from a sample of spherical latex particles of $700 \AA$ diameter in solution of $\mathrm{D}_{2} \mathrm{O}$. For both TOF and monochromatic modes the same total measurement time of $45 \mathrm{~min}$ has been allocated for scattering, background and transmission measurements in order to enable a fair comparison of the data measured in the two modes with sufficient statistical quality. The monochromatic measurements at $10 \AA$ were made on the same sample in two configurations: a low- $q$ setting from 0.0015 to $0.04 \AA^{-1}$ with collimation $12.8 \mathrm{~m}$, rear

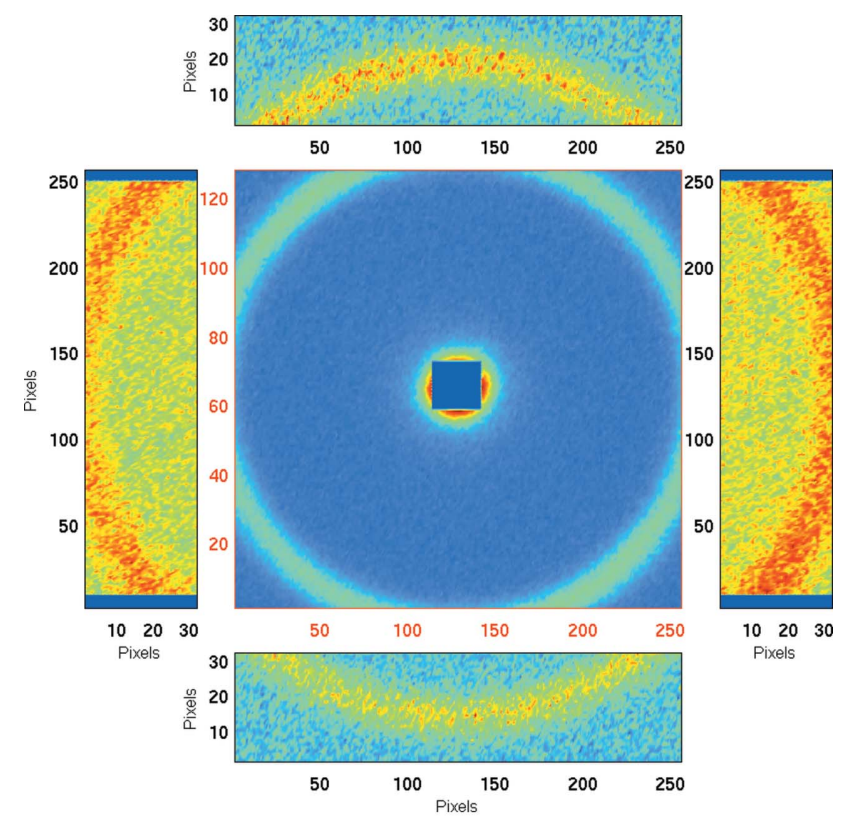

(a)

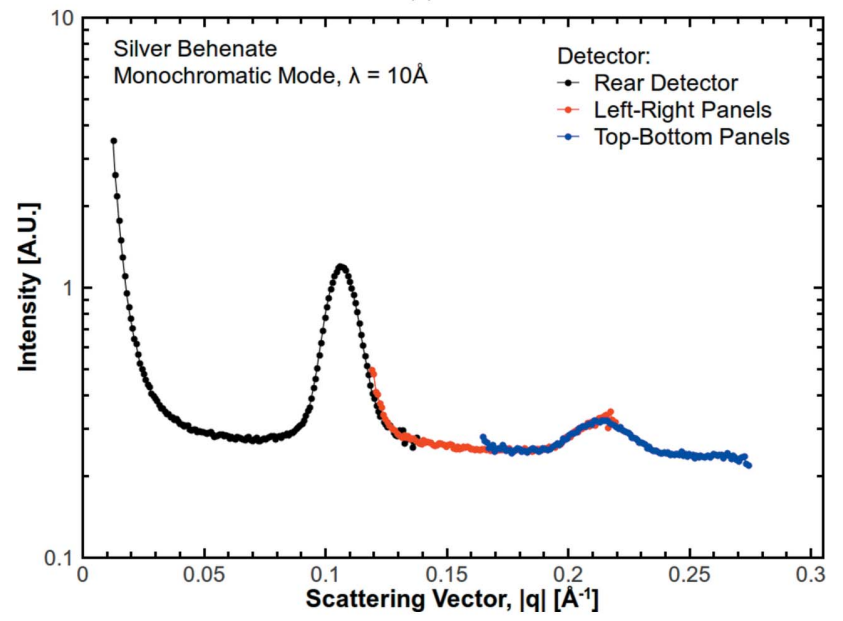

(b)

Figure 7

Small-angle scattering from silver behenate powder, which exhibits Debeye-Scherrer rings at $q=0.1076 \AA^{-1}$, corresponding to a layer spacing of $58.38 \AA$. (a) A two-dimensional representation of the five panels of multidetectors on D33 at distances of $2 \mathrm{~m}$ (rear), $1.2 \mathrm{~m}$ (top and bottom) and $1.39 \mathrm{~m}$ (left and right). Data for each panel are shown on its own ( $\log$ ) colour scale. (b) Regrouped I versus $q$ average for the rear, top, bottom, left and right detector panels covering a $q$ range from 0.011 to $0.282 \AA^{-1}$, and giving a dynamic $q$ range of $\sim 25$. detector $12.8 \mathrm{~m}$ and front detector $6.4 \mathrm{~m}$ (6.58 m); and a high- $q$ setting from 0.009 to $0.2 \AA^{-1}$ using collimation $2.8 \mathrm{~m}$, rear detector $2.4 \mathrm{~m}$ and front detector $1.2 \mathrm{~m}(1.38 \mathrm{~m})$. In both monochromatic configurations, the front detectors are situated at approximately half the distance of the rear detector, and as such the panels have been moved to a half-closed position such that the angular size of the front detector aperture matches that of the rear detector. Once more, all data have been treated as described in $\S 3$. It should be noted that, in the overlap region of the two monochromatic configurations, the systematically higher and less structured lowest $q$ data measured in configuration 2 (green curve) compared with the highest $q$ data measured in configuration 1 (red curve) are due to the rather different angular resolution between the two configurations. This has the effect of smoothing scattering features over the scattering vector resolution $\Delta q$ and presenting an apparently higher intensity than the higherresolution data.

The TOF measurements were made with the rear detector at $10 \mathrm{~m}$ and front panels at $4 \mathrm{~m}$ (horizontal) and $4.185 \mathrm{~m}$ (vertical) from the sample and in the fully open position. Choppers $\{1\}$ and $\{2\}$ defined a wavelength resolution of $15.6 \%$ on the rear detector and $20.1 \%$ on the front panels. Fig. 8 shows the intensity as a function of $q$ for the TOF data regrouped from 100 time channels within a wavelength range from 1.3 to $18 \AA$. The data clearly show oscillations corresponding to the form factor of the rather monodisperse latex particles in solution and cover a total $q$ range from 0.001 to $0.6 \AA^{-1}$, i.e. a dynamic $q$ range of 600 in a single measurement. Noisy data points at low and high $q$ extremities arise from the fact that only the ends of the wavelength band contribute to the data. The form factor oscillations are more pronounced for the low- $q$ monochromatic data, because of the better wavelength resolution of $10 \%$ compared with the relaxed TOF conditions. In contrast, for the monochromatic high- $q$ setting the feature is not resolved owing to a lack of angular resolution. On the other hand, the TOF mode eliminates the need to

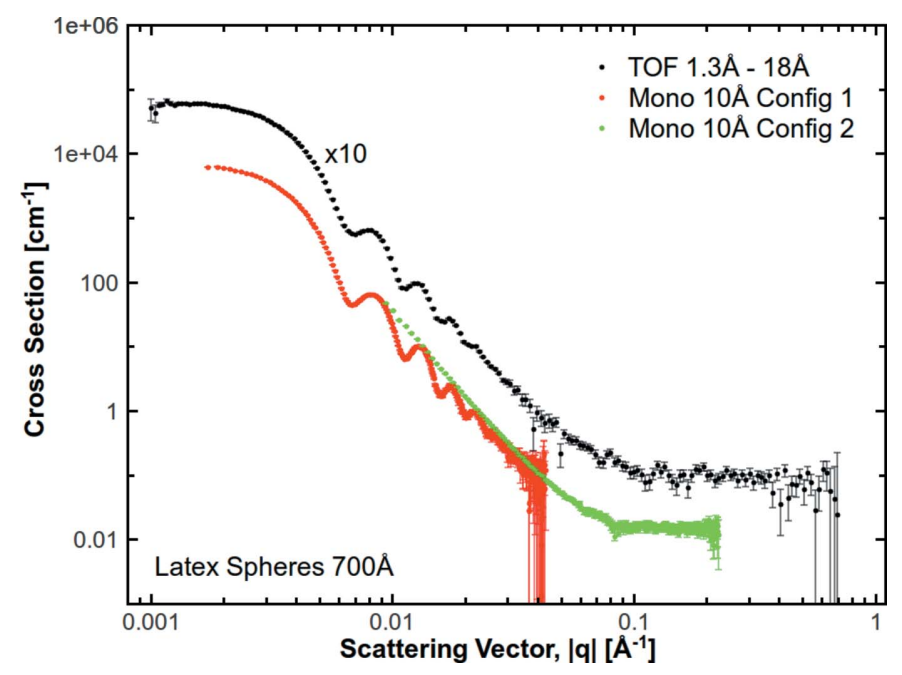

Figure 8

Small-angle scattering from $700 \AA$ spherical latex particles in $\mathrm{D}_{2} \mathrm{O}$ solution, measured in both monochromatic and TOF modes on D33. 
measure the same sample at different instrument configurations in order to generate a scattering curve over the full accessible $q$ range.

\section{Conclusions}

Small-angle neutron scattering has become an important tool in fields of research as diverse as soft-matter science, biology, physics, magnetism and materials science. This is apparent from the consistent high demand for SANS beam time, with an overload factor of three for the SANS suite at ILL. As a consequence, D33 is the third SANS instrument to be built at ILL, fully entering the user programme in 2013. Specific emphasis was made in the specification of D33 on its versatility and flexibility in terms of resolution, dynamic $q$ range, monochromatic and time-of-flight modes of operation, provision of polarization and analysis options, and flexibility and space in the sample zone, in an attempt to anticipate the future needs of the SANS community of users. The time-offlight mode of operation on a continuous reactor neutron source is a novel feature and allows both flexible wavelength resolution and a very wide dynamic $q$ range, easily tailored to the specific scientific case. Two large multitube detectors extend the dynamic $q$ range further, giving $q_{\max } / q_{\min }=25$ in monochromatic mode and a very large $q_{\text {max }} / q_{\text {min }}>1000$ in TOF mode. The high neutron flux and the flexibility of its setup make D33 suited for real-time experiments and weakly scattering samples. The polarization and analysis options facilitate and expand studies in magnetism. D33 is sufficiently flexible to allow various non-standard configurations, such as multi-beam measurements to extend the low- $q$ range or for small samples, while retaining sufficient intensity for scanning neutron imaging measurements resolving both transmission and scattering contrast mechanisms. A final possibility, not explored here, is the combination of both the monochromatic and TOF modes of operation to allow a more quantitative and direct measurement of inelastic scattering effects. The position of D33 and the open and flexible layout of the sample zone are such as to allow large and bulky sample environments and high magnetic fields. With the enormous flexibility of D33, the main challenge for users of the instrument is to find the optimum experimental configuration and approach for a particular scientific problem. This article is intended to give some general pointers as to the capabilities and possibilities offered by this latest SANS instrument at ILL.

\section{Acknowledgements}

The authors thank the members of the LSS group and the numerous ILL directors, as well as the SANS user community, for their support of this project. We gratefully acknowledge the reliable service given by ILL technical staff during the development phase and in the daily operation of D33. In particular, we would like to thank Peter Timmins, Ken Andersen, Anna Sokalova, Martin Platz, Bruno Guerard and Paolo Mutti for fruitful discussions, and Pierre Courtois and
Thierry Bigault for the provision of mirrors for the wavelength cut-off filters and polarizers.

\section{References}

Bazhenov, A. N., Lobashev, V. M., Pirozhkov, A. N. \& Slusar, V. N. (1993). Nucl. Instrum. Methods Phys. Res. Sect. A, 332, 534-536.

Bigault, T., Delphin, G., Vittoz, A., Gaignon, V. \& Courtois, P. (2014). J. Phys. Conf. Ser. 528, 012017.

Bonini, M., Wiedenmann, A. \& Baglioni, P. (2007). J. Appl. Cryst. 40, s254-s258.

Campbell, R. A., Wacklin, H. P., Sutton, I., Cubitt, R. \& Fragneto, G. (2011). Eur. Phys. J. Plus, 126, 107.

Cubitt, R. \& Fragneto, G. (2002). Appl. Phys. Mater. Sci. Process. 74, s329-s331.

Cubitt, R., Schweins, R. \& Lindner, P. (2011). Nucl. Instrum. Methods Phys. Res. Sect. A, 665, 7-10.

Dewhurst, C. D. (2008). Meas. Sci. Technol. 19, 034007.

Dewhurst, C. D. (2012). Nucl. Instrum. Methods Phys. Res. Sect. A, 683, 16-23.

Dewhurst, C. D. (2014). J. Appl. Cryst. 47, 1180-1189.

Disch, S., Wetterskog, E., Hermann, R. P., Wiedenmann, A., Vainio, A., Salazar-Alvarez, G., Bergström, L. \& Brückel, Th. (2012). New J. Phys. 14, 013025.

Dufour, C., Fitzsimmons, M. R., Borchers, J. A., Laver, M., Krycka, K. L., Dumesnil, K., Watson, S. M., Chen, W. C., Won, J. \& Singh, S. (2011). Phys. Rev. B, 84, 064420.

Gilles, R., Keiderling, U. \& Wiedenmann, A. (1998). J. Appl. Cryst. 31, 957-959.

Grigoriev, S. V., Okorokov, A. I. \& Runov, V. V. (1997). Nucl. Instrum. Methods Phys. Res. Sect. A, 384, 451-456.

Grigoryeva, N. A., Grigoriev, S. V., Eckerlebe, H., Eliseev, A. A., Lukashin, A. V. \& Napolskii, K. S. (2007). J. Appl. Cryst. 40, s532-s536.

Grillo, I. (2008). Small-Angle Neutron Scattering and Applications in Soft Condensed Matter. Heidelberg: Springer-Verlag.

Heenan, R. K., King, S. M., Turner, D. S. \& Treadgold, J. R. (2006). Proceedings of ICANS XVII, 25-29 April 2005, Santa Fe, New Mexico, USA, p. 780.

Heenan, R. K., Penfold, J. \& King, S. M. (1997). J. Appl. Cryst. 30, 1140-1147.

Heenan, R. K., Rogers, S. E., Turner, D., Terry, A. E., Treadgold, J. \& King, S. M. (2011). Neutron News, 22(2), 19-21.

Heinemann, A., Hermann, H., Wiedenmann, A., Mattern, N. \& Wetzig, K. (2000). J. Appl. Cryst. 33, 1386-1392.

Hollamby, M. J. (2013). Phys. Chem. Chem. Phys. 15, 10566.

Holmes, A. T., Walsh, G. R., Blackburn, E., Forgan, E. M. \& SaveyBennett, M. (2012). Rev. Sci. Instrum. 83, 023904.

Honecker, D., Ferdinand, A., Döbrich, F., Dewhurst, C. D., Wiedenmann, A., Gómez-Polo, C., Suzuki, K. \& Michels, A. (2010). Eur. Phys. J. B, 76, 209-213.

Huang, T. C., Toraya, H., Blanton, T. N. \& Wu, Y. (1993). J. Appl. Cryst. 26, 180-184.

Ishikawa, Y., Furusaka, M., Niimura, N., Arai, M. \& Hasegawa, K. (1986). J. Appl. Cryst. 19, 229-242.

James, M., Nelson, A., Brule, A. \& Schulz, J. C. (2006). J. Neutron Res. 14, 91-108.

Kampmann, R., Haese-Seiller, M., Kudryashov, V., Nickel, B., Daniel, C., Fenzl, W., Schreyer, A., Sackmann, E. \& Rädler, J. (2006). Phys. B Condens. Matter, 385-386, 1161-1163.

Krycka, K. L., Booth, R. A., Hogg, C. R., Ijiri, Y., Borchers, J. A., Chen, W. C., Watson, S. M., Laver, M., Gentile, T. R., Dedon, L. R., Harris, S., Rhyne, J. J. \& Majetich, S. A. (2010). Phys. Rev. Lett. 104, 207203.

Krycka, K., Borchers, J., Ijiri, Y., Booth, R. \& Majetich, S. (2012). J. Appl. Cryst. 45, 554-565.

Laver, M., Forgan, E. M., Brown, S. P., Charalambous, D., Fort, D., Bowell, C., Ramos, S., Lycett, R. J., Christen, D. K., Kohlbrecher, J., Dewhurst, C. D. \& Cubitt, R. (2006). Phys. Rev. Lett. 96, 167002. 
Laver, M., Mudivarthi, C., Cullen, J. R., Flatau, A. B., Chen, W.-C., Watson, S. M. \& Wuttig, M. (2010). Phys. Rev. Lett. 105, 027202.

Mühlbauer, S., Binz, B., Jonietz, F., Pfleiderer, C., Rosch, A., Neubauer, A., Georgii, R. \& Böni, P. (2009). Science, 323, 915-919.

Petoukhov, A. K., Guillard, V., Andersen, K. H., Bourgeat-Lami, E., Chung, R., Humblot, H., Jullien, D., Lelievre-Berna, E., Soldner, T., Tasset, F. \& Thomas, M. (2006). Nucl. Instrum. Methods Phys. Res. Sect. A, 560, 480-484.

Ramazanoglu, M., Laver, M., Ratcliff, W., Watson, S. M., Chen, W. C., Jackson, A., Kothapalli, K., Lee, S., Cheong, S.-W. \& Kiryukhin, V. (2011). Phys. Rev. Lett. 107, 207206.
Rastovski, C., Dewhurst, C. D., Gannon, W. J., Peets, D. C., Takatsu, H., Maeno, Y., Ichioka, M., Machida, K. \& Eskildsen, M. R. (2013). Phys. Rev. Lett. 111, 087003.

Seeger, P. A. \& Hjelm, R. P. (1991). J. Appl. Cryst. 24, 467-478.

Ueland, B. G., Lynn, J. W., Laver, M., Choi, Y. J. \& Cheong, S.-W. (2010). Phys. Rev. Lett. 104, 147204.

Wagner, V., Friedrich, H. \& Wille, P. (1992). Phys. B Condens. Matter, 180-181, 938-940.

Well, A. A. van (1992). Phys. B Condens. Matter, 180-181, 959-961. Wismayer, M. P., Lee, S. L., Thomson, T., Ogrin, F. Y., Dewhurst, C. D., Weekes, S. M. \& Cubitt, R. (2006). J. Appl. Phys. 99, 08 E707. 